\title{
A New Adaptive Current Protection Scheme for Distributed Systems with Distributed Generation
}

\author{
Wanxing Sheng ${ }^{1, a}$, XiaoLi Meng ${ }^{1, b}$ and Jing $\mathrm{Ma}^{2, \mathrm{c}}$ \\ ${ }^{1}$ Power Distribution Department, China Electric Power Research Institute, Beijing, 100192, China \\ ${ }^{2}$ State Key Laboratory of Alternate Electrical Power System with Renewable Energy Sources, \\ North China Electric Power University, Beijing, 102206, China \\ a hdmajing@ncepu.edu.cn, ${ }^{b}$ hdmajing@163.com, ${ }^{c}$ hamajing@aliyun.com.cn
}

\begin{abstract}
Keywords: Distributed generation (DG), adaptive protection, distribution communication,
\end{abstract} distributed systems

\begin{abstract}
An adaptive protection scheme using digital relays is proposed for the protection of the distributed systems. Firstly, the impact of distributed generators on protection coordination is discussed. Then, an adaptive protection scheme is proposed for distributed systems with distributed generation (DG). Simulated results on an actual distribution system show that the proposed approach can perform adaptive primary and backup protection functions under both balanced and unbalanced fault conditions. Also, the proposed approach is adaptive to the fault types as well as capacities of DG. Compared with the traditional current protection scheme, the primary and backup protection regions have been extended considerably.
\end{abstract}

\section{Introduction}

Deregulation and growth of competitive supply markets have recently increased the interest in distributed generation (DG) from renewable and traditional (but highly efficient) sources. The growing concern on climate changes and the always-present oil crisis also multiplies this interest. Furthermore, introduction of DG to distribution networks would cost less than expansion of transmission and distribution facilitates [1]. Studies have predicted that a presence of approximately $20 \%$ on energy sources for the years 2010 to 2020 [2]. This means that DG would feed loads around its location and the assumption of the distribution system being radial is not likely to hold in the near future. One would then be looking at a multisource system.

For a multisource system, protection devices are necessary to be directional sensitive [3-5]. In addition, connecting a generator to a distribution network has the effect of increasing the fault current. Reference [6] demonstrates that fault currents measured by the relays will vary after DGs are connected. Thus, new fault current and setting should be calculated for the protection coordination preparation. However, the protection coordination under the changing system condition (DG's capacity) is a time-consuming and a tedious task. Thus, disconnection of all DGs from distribution systems is the current local distribution companies' practice during fault in order to maintain the original relay coordination. But throwing off all DGs from system every time a temporary fault occurs would make the system very unreliable. To overcome disconnecting all downstream DGs, An adaptive protection scheme was presented in [7], which depends on remote communication capabilities. Reference [8] proposes a microgrid protection using communication network. Communication is a major activity in an adaptive relaying system. However, traditional protection relay coordination relies on standalone units that use local measurements and settings as the basis for the decision making. Communication plays a very limited role in these legacy systems. A new era of decentralized control and efficiency demands has led to an environment demanding efficiency and reliability that pushes these legacy methods to their limits. In this paper, the impact of DG on protection coordination is first analyzed. Then a comprehensive adaptive protection scheme is proposed for distribution systems with a few larger DGs that would undermine the system reliability. In the proposed scheme, communication will play an important role to provide 
more information for the relay coordination besides the relay settings. The validity and effectiveness of the proposed scheme have been demonstrated by PSCAD/EMTDC.

\section{Adaptive Protection Scheme}

\subsection{Communication Coordination and General Scheme}

To avoid throwing off all DGs from system every time, the relays should respond to the changing system conditions and adapt according to the new prevailing conditions. In adaptive coordination, communication is a major activity in an adaptive relaying system. Considering the operating cost and the connection speed, wire- or fiber-based systems are viable alternatives for communication of data in adaptive schemes [9]. The costs can be further controlled by not using specific communication channels, but by existing connections already deployed in that part of the system. For instance, if 'smart grid' technologies have already been deployed, the corresponding communication channel can be used.

In this situation, the relay at the bus with a communication link to the adjacent or downstream measurements of the current and switching device status is able to perform adequately for faults encountered in distribution systems and enable cost-effective protection schemes. Each relay has an adaptive primary function and an adaptive backup function. If a fault occurs within the range of the primary function zone, currents of loads and DG units backward of the relay are measured in real time, and then are sent to the relay. The relay will identify the fault using the Thevenin equivalent model and isolate the faulted zone by tripping appropriate breakers. If a fault occurs within the range of the backup function zone, currents of loads and DG units forward of the relay are transformed to branch currents and then the dramatic changes caused by DG units and loads can be eliminated. The following subsections explain the scheme in detail.

\subsection{Adaptive Primary Protection Setting for Feeders with DGs}

For traditional distribution systems, the substation is the only source of power. In addition, due to the substations away from large generation units, the initial high "subtransient component" does not exist in the fault current transients. Assume that the faulted phase can be determined previously, the fault current can be approximated by its steady-state value. In this way, the feeder can be represented by a steady-state model, in which the substation is represented as a voltage source behind a Thevenin impedance. If there are conventional DGs on the feeder, the above feeder model can be obtained easily by employing the simple Thevenin equivalent models for these generators [10]. However, for other kinds of DGs that response considerably after a fault occurs, the same technique can not be applied [11]. Therefore, a new scheme is needed to incorporate all DGs into the fault analysis.

DG1 can be represented as an injected phase current $i_{1}$, the lines CD and DE are represented by their series impedance $z_{C E}$, and the Load 3 can be represented by its equivalent impedances $z_{d 3}$. The corresponding equivalent circuit of these devices can then be represented by Norton equivalent model as shown in Fig. 1, which can then be easily transformed to Thevenin equivalent model as shown in Fig. 2 . The voltage $u_{1}$ behind the Thevenin impedance is calculated as:

$$
u_{1}=i_{1} \frac{z_{C E} z_{d 3}}{z_{C E}+z_{d 3}} \text {. }
$$

Thus, the adaptive primary protection setting for $\mathrm{R} 4$ can be formulated as:

$$
\begin{aligned}
& i_{p s 4}=\frac{k_{k} k_{d} u_{1}}{z_{m}+z_{l}} . \\
& Z_{m}=\frac{Z_{C E} Z_{d 3}}{Z_{C E}+Z_{d 3}} .
\end{aligned}
$$


where $i_{p s 4}$ is the adaptive primary protection setting for R4. $z_{m}$ is the integrated impedance of source side. $z_{l}$ is the protected line impedance. $k_{d}$ is the coefficient of fault type and can be previously determined by software used by utilities. $k_{k}$ is the coefficient of reliability.

A similar procedure can also be applied to R5 for its adaptive primary protection setting $i_{p s 5}$.

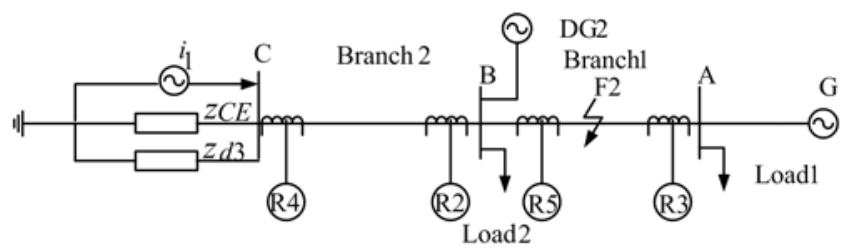

Fig.1. Norton equivalent model behind R4.

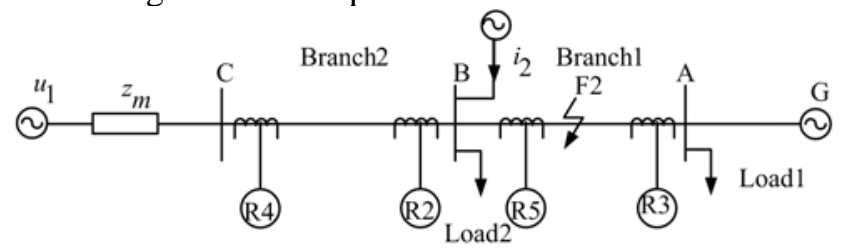

Fig.2. Thevenin equivalent model behind R4.

\subsection{Adaptive Backup Protection Setting for Feeders with DGs}

In the event of a switching device failure, the next upstream device, or device combination, must operate to provide backup protection. For F2, if the measured current of R5 is still above the threshold after a certain time delay, measurements of the current (including the injected phase current of DG2, $i_{2}$ ) and switching device status at bus B will be sent to R4 to provide backup protection. However, since DG2 may response considerably after a fault occurs, measured currents of R4 will change dramatically.

To eliminate the effect of DG2 on these measurements, a novel method is proposed as follows.

Firstly, DG units are represented as injected currents. The nodal equations of the distribution systems can be written as:

$$
\begin{aligned}
& \mathbf{I}_{\mathbf{N}}=\mathbf{Y}_{\mathbf{N}} \mathbf{U}_{\mathbf{N}} . \\
& \mathbf{Y}_{\mathbf{N}}=\mathbf{A Y A}^{\mathbf{T}} .
\end{aligned}
$$

where $\mathbf{Y}_{\mathbf{N}}$ is the node admittance matrix. $\mathbf{U}_{\mathbf{N}}$ is the voltage at each node. $\mathbf{I}_{\mathbf{N}}$ is the current injected at each node. $\mathbf{A}$ is the node correlation matrix. $\mathbf{Y}$ is the branch admittance matrix.

Then, the relationship between the branch current $\mathbf{I}_{\mathbf{B}}$ and the node voltage $\mathbf{U}_{\mathbf{N}}$ can be expressed as:

$\mathbf{I}_{\mathrm{B}}=\mathbf{Y} \mathbf{A}^{\mathrm{T}} \mathbf{U}_{\mathbf{N}}$.

From (4)-(6), the relationship between the branch current $\mathbf{I}_{\mathbf{B}}$ and the injected current $\mathbf{I}_{\mathbf{N}}$ can be deduced as:

$\mathbf{I}_{\mathbf{B}}=\mathbf{Y A}^{\mathrm{T}} \mathbf{Y}_{\mathbf{N}}^{-1} \mathbf{I}_{\mathbf{N}}$.

where $\mathbf{C}(\lambda)=\mathbf{Y} \mathbf{A}^{\mathbf{T}} \mathbf{Y}_{\mathbf{N}}^{-1}$ is defined as the system correlation coefficient matrix.

Furthermore, the current of the $k^{\text {th }}$ branch caused by the injected current of DG2 is calculated as:

$i_{k, B}=\lambda_{k 2} i_{2}$.

where $i_{k, B}$ is the current of $k^{\text {th }}$ branch caused by the injected current of DG2. $\lambda_{k 2}$ is the element of the system correlation coefficient matrix $\mathbf{C}(\lambda)$.

Finally, the impact of DG2 on the of $k^{\text {th }}$ branch can be eliminated by:

$i_{k, d}=i_{k, m}-i_{k, B}$.

where $i_{k, m}$ is the measured current of $k^{\text {th }}$ branch. $i_{k, d}$ is the current of $k^{\text {th }}$ branch without the impact of DG2.

After the method is applied to R4 and R5, the measured currents of two relays will not be affected by DG2. In this situation, the adaptive backup protection setting can be formulated as: 
$i_{b s 4}=k_{k}^{\prime} i_{p s 5} / k_{b}$.

$k_{b}=i_{1, d} / i_{2, d}$.

where $i_{b s 4}$ is the adaptive backup protection setting for R4. $i_{p s 5}$ is the primary protection setting for R5. $k_{b}$ is the branch coefficient. $k_{k}^{\prime}$ is the coefficient of reliability. $i_{1, d}$ is the current of branch 1 without the impact of DG2. $i_{2, d}$ is the current of branch 2 without the impact of DG2.

In (10), DG units do not have any effect on the adaptive backup protection setting.

\section{Test Systems and Results}

\subsection{Test Systems}

To test the performance of the proposed adaptive protection method, a practical $10 \mathrm{kV}$ distributed system in Tianjin power network is used in this study as shown in Fig. 3. The base capacity is 500MVA, and the base voltage is $10.5 \mathrm{kV}$. DG1 and DG2 with P-Q control schemes are connected to bus $\mathrm{C}$ and bus E, respectively. Their nominal capacities are both 10MVA. Branches AB, BC and $\mathrm{AF}$ are all overhead lines. The resistance and reactance of these lines are $r_{1}=0.27 \Omega / \mathrm{km}$ and $x_{1}=0.347 \Omega / \mathrm{km}$, respectively. Moreover, branches CD, DE and FG are all underground cables. The resistance and reactance of these cables are $r_{1}=0.259 \Omega / \mathrm{km}$ and $x_{1}=0.093 \Omega / \mathrm{km}$, respectively. The nominal capacity and the nominal power factor of each load are 6MVA and 0.85, respectively. Additionally, any lines that will experience bidirectional current flow will need two protection devices at each end of line, whereas any lines that will not experience bidirectional current flow will only need one protection device instead of two, thus reducing the cost. The devices at both ends of line are capable of sensing the fault with different current directions. The test system is simulated by using PSCAD/EMTDC.

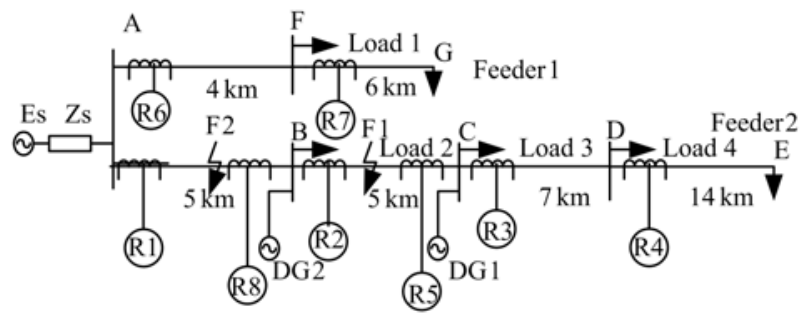

Fig.3. A practical $10 \mathrm{kV}$ distribution system in Tianjin power network.

In the following analysis, R8 and R5 are used as an example to demonstrate the effectiveness and accuracy of the adaptive primary function and the adaptive backup function, respectively. $I_{p s 5}$ and $I_{p m 5}$ represent the adaptive primary protection setting and the measured current of R5, respectively. $I_{p s 8}$ and $I_{p m 8}$ represent the adaptive primary protection setting and the measured current of R8, respectively. $I_{b s 5}$ and $I_{b m 5}$ represent the adaptive backup protection setting and the measured current of R5 without the impact of DG2, respectively.

\subsection{Responses of Adaptive Primary Protection System}

For conventional source in the distribution systems, assume that the fault type can be determined previously, the fault current can be approximated by its steady-state value. But for DGs that response considerably after a fault occurs, the same technique can not be applied. Therefore, the proposed adaptive protection scheme mainly focuses on lines fed through DGs. A three-phase fault was applied to the middle of the line between buses B and C at 0.30 seconds. The fault is cleared after 0.5 seconds. When the fault occurs, the fault current flowing through R5 is fed by DG1. Thevenin equivalent model behind R5 can be obtained by using (1) and (3), and $I_{p s 5}$ can be calculated by using (2). Fig. 4 shows the curves of $I_{p s 5}$ and $I_{p m 5}$. It can be found that after fault occurs, $I_{p m 5}$ responds considerably and becomes larger than $I_{p s 5}$. Thus, the primary protection issues a signal to switching device to trip fault. 


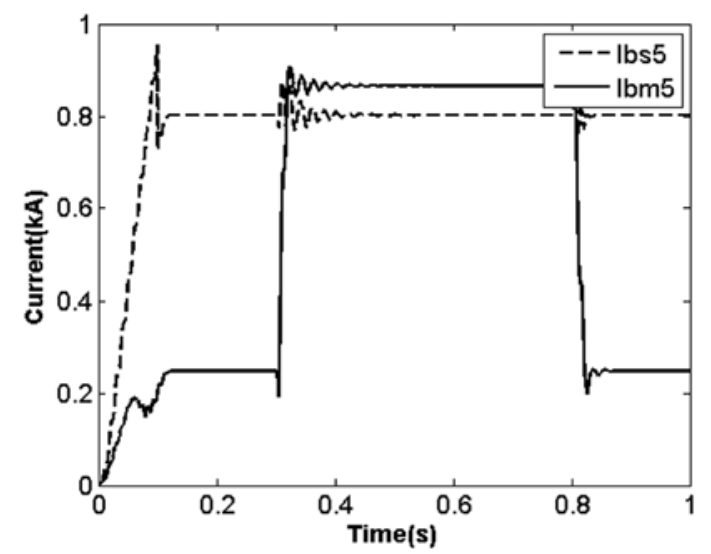

Fig.4. The adaptive primary protection setting and the measured current of R5 when a three-phase fault occurs in the middle of Section BC.

\subsection{Responses of Adaptive Backup Protection System}

If a fault occurs on Section AB close to bus $B$, and the measured current of R8 is still above the threshold after a certain time delay, then R5 must operate to provide adaptive backup protection. In this situation, the impact of DG2 on the branch currents is first eliminated by using (4)-(9). Then, $I_{b s 5}$ is calculated by using (10) and (11). Fig. 5 shows $I_{b s 5}$ and $I_{b m 5}$ in case of a three-phase fault. It can be found that no matter what types of faults occur, $I_{b m 5}$ is greater than $I_{b s 5}$ in both figures. Thus, in each case, the adaptive backup protection at R5 will issue a signal to the switching device to clear the fault on the adjacent Section AB.

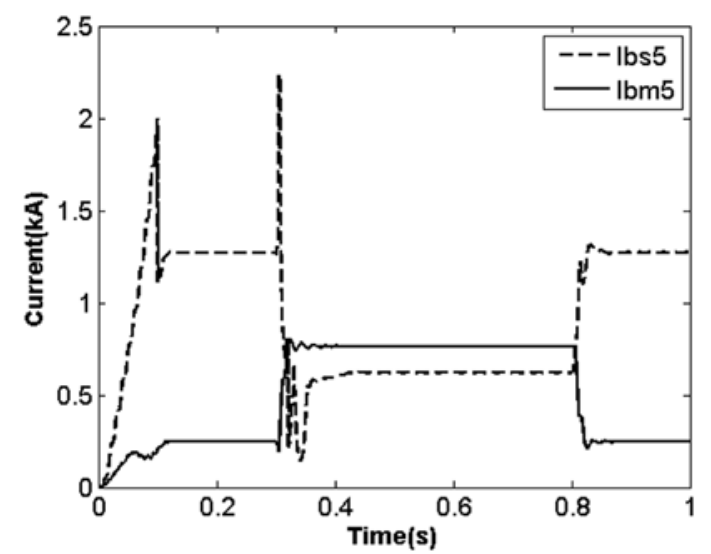

Fig.5. The adaptive backup protection setting and the measured current of R5 when a three-phase fault occurs on Section AB close to bus B.

A comprehensive fault analysis is performed for different locations on the system, different fault types (balanced and unbalanced), and different capacities of DG1. It can be concluded that the range of the adaptive primary protection is about $80 \%$ length of its own line. Furthermore, the adaptive backup protection is able to clear faults in the adjacent or downstream zones. However, the range of the conventional current primary protection is less than $20 \%$ length of its own line. In addition, the conventional current backup protection can not clear faults even in its own line. Furthermore, the proposed scheme is not affected by the fault types and capacities of DG1. Thus, the protection performance has been greatly improved.

\section{Conclusion}

One problem with distributed generation (DG) implementation is designing a proper protection scheme. After connecting DG, part of the system may no longer be radial, which means the traditional protection schemes might not work successfully. The adaptive scheme proposed here offers a practically acceptable solution to this problem in the distribution system. The proposed scheme is adaptive to the fault types as well as capacities of DG. Furthermore, the primary and 
backup protection regions have been extended considerably compared with the traditional current protection scheme. Simulation results verify that the proposed scheme is able to clear faults under both balanced and unbalanced fault conditions. With the connection status of DG and/or load varied, the system correlation coefficient matrix and the voltage behind the Thevnin impedance are modified. This part will be analyzed in a later paper.

\section{Acknowledgment}

This paper is supported by the Key Project of Science and Technology of State Grid Corporation of China (PD71-13-031).

\section{References}

[1] H. L. Willis and W. G. Scott: Distributed Power Generation Planning and Evaluation (Marcel Dekker, New York 2000).

[2] P. Barker and R. W. de Mello: Determining the impact of distributed generation on power systems: Part 1-Radial power systems, in Proc. IEEE Power Eng. Soc. Summer Power Meeting (2000), p. 1645-1658.

[3] M. A. Anthony: Electric Power System Protection and Coordination (McGraw-Hill, New York 1995).

[4] J. L. Blackburn: Protective Relaying Principles and Applications (Marcel Dekker, New York 1998).

[5] P. M. Anderson: Power System Protection(IEEE Press, New York 1999).

[6] N. Hadjsaid, J. Canard, and F. Dumas: Dispersed generation impact on distribution networks, IEEE Comput. Appl. Power, Vol. 12 (1999), p. 22-28.

[7] S. M. Brahma, A. A. Girgis: Development of adaptive protection scheme for distribution systems with high penetration of distributed generation, IEEE Trans. Power Delivery, Vol. 19 (2004), p. 56 - 63.

[8] Sortomme E., Venkata S.S., Mitra J: Microgrid Protection Using Communication-Assisted Digital Relays, IEEE Trans. Power Delivery, Vol. 25 (2010), p. 2789 - 2796.

[9] D. G. Hart, D. Novocel, M. Subramanian, and M. Ingram: Real-time wide area measurement for adaptive protection and control, in Proc. Nat. Sci. Found./Dept. of Energy/Elect. Power Res. Inst.-Sponsored Workshop on Future Res. Directions for Complex Interactive Electric Networks, Washington DC (2000).

[10]T. Chen et al.: Three-phase cogeneration and transformer models for distribution system analysis, IEEE Trans. Power Del., Vol. 6 (1991).

[11]J. C. Gomez and M. M. Morcos: Coordinating overcurrent protection and voltage sags in distributed generation systems, IEEE Power Eng. Rev., vol. 22 (2002), p. 16-19. 